This document is the accepted manuscript version of the following article:

Dejea, H., Tanner, C., Achanta, R., Stampanoni, M., Perez-Cruz, F., Konukoglu, E., \& Bonnin, A. (2019). Synchrotron X-ray phase contrast imaging and deep neural networks for cardiac collagen quantification in hypertensive rat mode1. In $Y$. Coudière, $V$. Ozenne, E. Vigmond, \& N. Zemzemi (Eds.), Lecture notes in computer science: Vol. 11504. Funtcional imgaing and modeling of the heart. 10th international conference, FIMH 2019, Bordeaux, France, June 6-8, 2019, Proceedings. https:// doi .org/10.1007/978-3-030-21949-9_21

\title{
Synchrotron X-ray phase contrast imaging and deep neural networks for cardiac collagen quantification in hypertensive rat model
}

\author{
Hector Dejea ${ }^{1,2^{* \dagger}}$, Christine Tanner ${ }^{3 \dagger}$, Radhakrishna Achanta ${ }^{4}$, Marco Stampanoni $^{2}$, \\ Fernando Perez-Cruz ${ }^{4}$, Ender Konukoglu ${ }^{3 \neq}$ and Anne Bonnin ${ }^{1 \neq}$ \\ ${ }^{1}$ Paul Scherrer Insitut, Villigen PSI, Switzerland \\ ${ }^{2}$ Institute for Biomedical Engineering, University and ETH Zürich, Zürich, Switzerland \\ ${ }^{3}$ Computer Vision Laboratory, ETH Zürich, Zürich, Switzerland \\ ${ }^{4}$ Swiss Data Science Center, Lausanne, Switzerland \\ * hector.dejea@psi.ch \\ $\dagger \ddagger$ Both authors contributed equally to this work.
}

\begin{abstract}
An excessive deposition of collagen matrix in the myocardium has been clearly identified as an indication of the progression towards heart failure. Nevertheless, few studies have been performed for its quantification and most of them use 2D histological images, thus losing valuable encoded 3D information. In this study, several biopsies of areas of the left ventricle from age-matched spontaneously hypertensive rats and Wistar Kyoto rats were imaged using synchrotron radiation-based X-ray phase contrast imaging. Then, an optimized deep neural network was used for automatic image segmentation in order to assess collagen fraction differences between models as well as its age dependency. The results show a general increase in the collagen percentage in the hypertensive model and for older rats. Such tendency is comparable with the reports found in the literature. Therefore, this proof of concept shows that synchrotron imaging in combination with deep neural networks is a powerful tool for the investigation and quantification of cardiac microstructures.
\end{abstract}

Keywords: Myocardial collagen fraction, Synchrotron imaging, Deep neural network, Image segmentation.

\section{Introduction}

Cardiovascular diseases (CVDs) are the leading cause of mortality, being responsible for $31 \%$ of the deaths worldwide [1]. Particularly, remodelling CVDs cause structural changes in the heart and vasculature at all scales, from organ- to cell-level.

The cardiac tissue (myocardium) is primarily composed of muscle cells called cardiomyocytes. They are arranged in spatially-oriented fibre-like structures (myofibers) and provide the contractile function of the heart under the electrical stimulus transmitted by pacemaker cells [2]. 
In the myocardial extracellular space, a collagenous matrix serves as a scaffold, helping the alignment of the cardiomyocytes. This matrix also avoids overstretching of cardiomyocytes and thus, ensures their integrity, among other functions. It is mainly composed of type 1 fibrillar collagen and maintained by specialized cells called myofibroblasts. Such matrix is organized in three different layers: endomysial collagen is found around single cardiomyocytes, perymysial collagen surrounds myofibers and epimysial collagen is located around groups of myofibres [3].

The collagen matrix is known to be affected by aging and remodelling disorders such as hypertension, myocardial infarction or transplant rejection. In these situations, the activity of the myofibroblast is increased and, consequently, an increased matrix deposition and fibrosis formation is observed. This situation causes myocardial stiffening and contractile dysfunction, thus eventually leading to heart failure syndrome [4].

Even if the relationship between collagen matrix remodelling and development of heart failure is known, the direct quantification of collagen amounts and its association with disease progression has not been extensively reported. Mainly, several quantification methods have been developed for 2D histological slices [5], which lack the spatial information and field of view that can be achieved with 3D imaging techniques, and suffer from artifacts due to slice preparation, such as tearing.

The main contribution in this topic was given by LeGrice et al. [6], who compared spontaneously hypertensive rats (SHR) and control Wistar Kyoto (WKY) to quantify the age-dependent deposition of collagen in the left ventricular lateral wall. Tissue blocks were prepared (including tissue processing, embedding and staining) and imaged in 3D using confocal microscopy, and collagen fraction was computed using dedicated image processing.

Alternatively, a recent preliminary study [7] proposed synchrotron radiation-based $\mathrm{X}$-ray phase contrast imaging (X-PCI) as a powerful methodology for the quantification of collagen amounts, thanks to its $3 \mathrm{D}$ high resolution capabilities and the exploitation of the tissue's change of index of refraction for contrast generation. This study quantified the collagen fraction in the left ventricular basal septum, lateral wall and apex for 3 months old control WKY rats using a semi-automatic machine learning method.

In this paper, we extend this study by including the SHR model and 12 months old specimens, so that differences between models as well as age dependency can be assessed. In addition, we make use of an optimized deep neural network for automatic collagen fraction calculation, such that datasets without any user inputs can be included in our analysis.

\section{$2 \quad$ Materials and Methods}

\subsection{Sample preparation}

Animal care and experimentation was performed in accordance with the European Union (Directive 2010/63/UE) and Spanish guidelines (RD 53/2013) for the use of experimental animals. Approval was received from the local animal experimentation ethics committee (CEEA 68/5435 and CEEA OB533/16). 
The presented study was performed on endomyocardial biopsies $\left(\sim 2 \times 2 \times 4 \mathrm{~mm}^{3}\right)$ of the basal septum, lateral wall and apex of the left ventricle in four different rats two 3 months old and two 12 months old WKY, and four age-matching SHR.

The samples were fixed in $4 \%$ paraformaldehyde and placed in specifically designed sample holders, mainly consisting of a thin-walled borosilicate glass tubes with an inner diameter of $2 \mathrm{~mm}$ (Fig. 1a).

\subsection{Data Acquisition and Reconstruction}

The synchrotron radiation-based X-ray tomography experiments were performed at the TOMCAT beamline (X02DA) of the Swiss Light Source (Paul Scherrer Institut, Villigen, Switzerland). Propagation-based X-PCI at an energy of $20 \mathrm{keV}$, propagation (sample-to-detector) distance of $20 \mathrm{~cm}$ and $0.65 \mu \mathrm{m}$ voxel size was used for image acquisition of the previously described cardiac biopsies. The biopsies were larger than the available field of view $\left(1.6 \times 1.44 \mathrm{~mm}^{2}\right)$, so three overlapping volumes from each biopsy were obtained. The volumes were acquired by a PCO.Edge 5.5 sCMOS Camera detector after conversion of X-rays to visible light with a LuAG:Ce $20 \mu \mathrm{m}$ scintillator (Fig. 1b). For each volume, 2501 projections over 180 degrees, 20 darks and 50 flats were measured with an exposure time of $120 \mathrm{~ms}$, leading to $\sim 7$ minutes acquisition time per volume. The dark projections are acquired without beam exposure to correct for the electronic noise of the detector, whereas flat projections are acquired without sample, to compensate for non-uniformities in beam intensity and the optical components.

After acquisition, the sinograms for each volume were computed. Then, the tomograms were reconstructed using the Gridrec algorithm [8], which is a modified fast version of the typical filtered back projection algorithm thanks to a regridding process to resample the Fourier space from polar to Cartesian coordinates. Such reconstruction procedure was performed with and without application of the Paganin's single-distance phase retrieval method [9], which allows to retrieve the phase information of the image thanks to a projection filtering process determined by the $\delta / \beta$ ratio from the index of refraction, which was set to 56.9. Reconstruction times were approximately 2 and 6.5 minutes, without and with Paganin method, respectively.

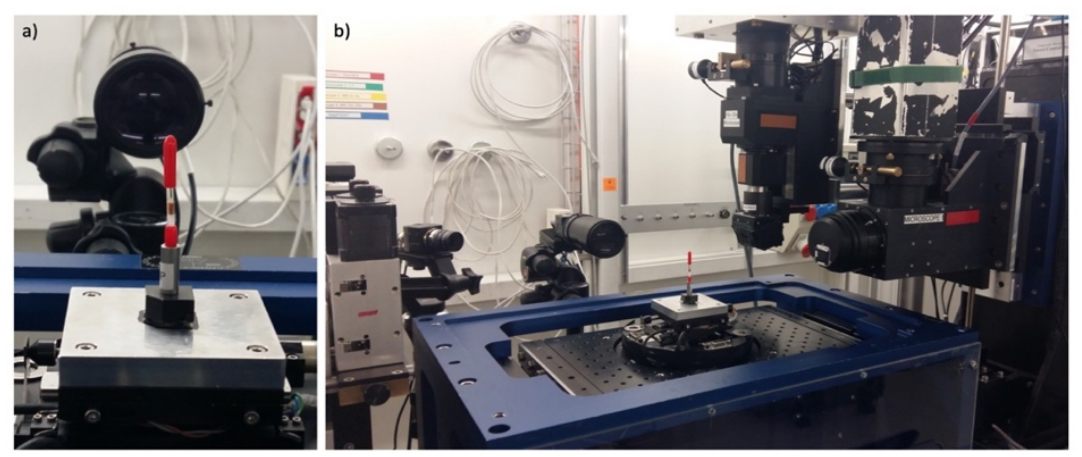

Fig. 1. a) Cardiac biopsy in a dedicated holder on the sample stage for acquisition. b) Overview of the synchrotron imaging setup. 


\subsection{Tissue segmentation}

Manual segmentation of the datasets was prohibitively time-consuming. Therefore, we first generated ground truth data from a subset of volumes using a well-established machine-learning based tool, which required manual interaction, then used it to train a neural network, which is later employed to segment the rest of the datasets.

Ground truth generation. As demonstrated in [7], ground truth data was generated with the open-source software Ilastik [10] and its interactive pixel classification module. Briefly, several regions of interest (of 460x460x460 voxels representing regions of $300 \times 300 \times 300 \mu \mathrm{m}^{3}$ ) from the acquired datasets were cropped and loaded on Ilastik. Iterative supervised labelling for background, cells and collagen was performed in 3D. The phase retrieved images were used to segment cells and background due to an increased contrast resulting from the Paganin method, while the collagen was segmented from the non-retrieved images due to increased sharpness, which can be slightly lost by the application of the Paganin method. Later, objects smaller than 150 voxels were removed for noise reduction purposes and both masks were fused to generate the final segmentation. We could have used the Ilastik tool to segment all datasets, however, empirically we observed that this tool required many user interactions for a satisfactory segmentation. Instead, we used Ilastik to carefully segment a few datasets with userinteractions and used it as ground truth to train the algorithms described next.

U-Net for collagen quantification. We used a well-studied and widely-used deep convolutional neural network, called U-Net [11], for segmenting the images into background, collagen and cells. The U-Net was chosen as it has previously shown good performance in several segmentation challenges. We built a $2 \mathrm{D}$ network that takes as

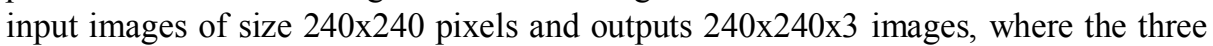
channels at the output are the class probabilities for each pixel, corresponding to the three classes: background, collagen and cells. Based on experience and previously proposed architectures, we designed a U-Net with 4 resolution levels in the encoding path that results in a bottleneck layer of size $15 \times 15$ with 512 channels (the architecture: input $>240 \times 240 \times 32>>120 \times 120 \times 64>>60 \times 60 \times 128>>30 \times 30 \times 256>>15 \times 15 \times 512)$. Each resolution level had two convolutional layers (kernel size of $2 \times 2$ with stride 1, followed by ReLU activation) and a max-pooling layer. The decoding path was formed of 4 resolution levels, which increased the size to $240 \times 240$ and reduced the channels to 3 in a symmetric way using transposed convolution with $4 \times 4$ kernels and stride 2 followed by ReLU activation. At the output, no non-linear activation was used, instead a soft-max layer converted the outputs to pixel-wise probabilities. Skip connections concatenated activations in the encoding path to the corresponding blocks in the decoding path.

Seven hyper-parameters (a-g) were optimized via an ablation study, where each time a single parameter is changed with respect to a reference setting. Next, we describe per parameter the two options, with the underlined first option being the reference setting. The input consisted of either (a0) a single slice or (a1) 3 neighbouring slices from (b0) 
only the non-retrieved image or (b1) both the non- and phase-retrieved image. The images were (c0) not augmented or (c1) augmented by 90, 180, 270 degrees rotations, horizontal or vertical flips. Optimization was based on (d0) the mean Dice coefficient or (d1) the logarithm of the mean Dice coefficient to cope with class imbalance [12], where Dice coefficient is defined as $D=2\left|S_{G T} \cap S_{U N e t}\right| /\left(\left|S_{G T}\right|+\left|S_{U N e t}\right|\right)$ with $|S|$ denoting the number of foreground ( $=1$ ) pixels of binary segmentation $S$. Regularization via setting weights to zero (dropout) was (e0) not enabled or (e1) with a probability of $50 \%$ performed. The U-Net consisted of (f0) 4 or (f1) 3 resolution levels. The U-Net decoder used (g0) transposed convolutions or (g1) bilinear upsampling followed by convolution. Furthermore, we compared the performance when increasing from $2.5 \%$ of the annotated data to $80 \%$.

The annotated data, which consisted of ten volumes of size 460x460x460 as described in the previous section, were randomly split into 6 training, 2 validation and 2 test volumes. A certain number of slices, e.g. $2.5 \%$ (11.5 slices), was then randomly selected from the training volumes for training the U-Net and from the validation volumes for determining training convergence (defined as no performance improvement over 1000 iterations for the validation data). To segment a volume, the trained U-Net was applied patch-wise with a stride length of 60 pixels and predicted class-probabilities were averaged. Ten U-Nets were trained based on different initial random initializations and randomly selected data splits to study gains from fusing their results and for getting results for all test cases. Fusion of the 1-4 results per manually segmented test case and of the 10 results for the other cases, was done by averaging per case, class and pixel the predicted probabilities from the U-Nets.

A supervised Gaussian Mixture Model (GMM) was used as baseline method. It clustered pixel intensities into 3 classes, based on model parameters (mean, standard deviation, mixture coefficients) learned from the ground truth segmentations of 8 volumes. It achieved a mean Dice coefficient of $66.3 \%$ in leave-2-volume-out tests. The correlation between the ground truth and GMM collagen fraction, measured by Pearson's correlation coefficient, was 0.17 .

The ablation study of the U-Net hyper-parameters, listed in (a-g) above, showed that options $\mathrm{c} 1, \mathrm{~d} 1, \mathrm{e} 1, \mathrm{f} 1, \mathrm{~g} 1$ improved mean Dice coefficient by 0.3 to $1.4 \%$ over the reference configuration and lead to a positive correlation between the ground truth and UNet collagen fraction $(0.17-0.43)$. Combining these 5 changes provided the highest correlation $(0.60)$. Using much more data $(80 \%)$ improved the mean Dice coefficient by $0.9 \%$ to $82.8 \%$ and the correlation to 0.73 . As the latter is a substantial improvement, we report here results for the U-Nets trained on $80 \%$ of the data.

Fig. 2 shows the segmentation result with the largest difference in collagen fraction to the ground truth $(3.4 \%$ vs. $1.0 \%)$. It can be observed that even for this worst result the U-Net segmentation is consistent across slices and appears reasonable. 

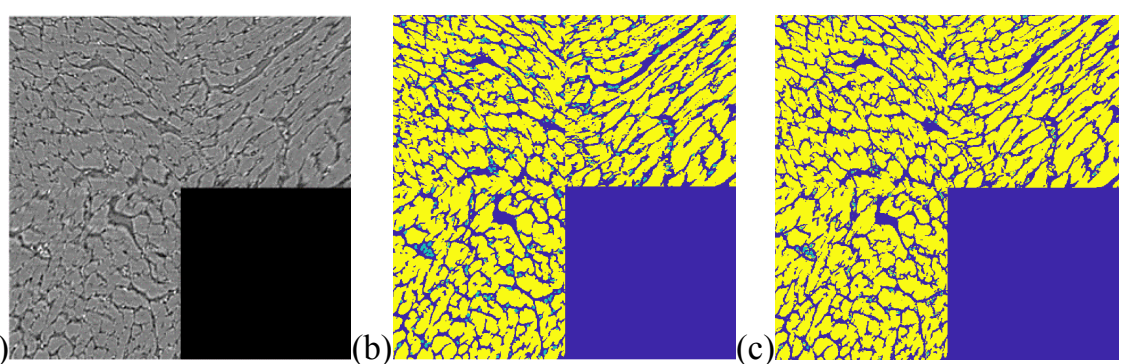

Fig. 2. Orthogonal central slices of (a) non-retrieved image volume (b) U-Net segmentation, and (c) ground truth for worst test result (Dice coefficient 75.2\%). Yellow represents myocytes, blue shows background and cyan depicts collagen. Slices are 460x460 pixels.

\section{Results}

The U-Net results of the collagen fraction in the different regions of WKY and SHR of 3 and 12 months old are summarized in Fig. 3. A general tendency towards higher collagen fractions can be observed for older rats as well as for the SHR case. Nevertheless, differences are observed when looking individually at each of the areas. Most of 3 months old measurements are located in a range between 2 and 7\%, while most 12 months old fractions are found between 5 and $9 \%$.

In the apex, there is an increased amount of collagen $(\sim 1 \%)$ in the SHR case both for 3 months old and 12 months old. In addition, the collagen fraction appears higher for older rats, being $\sim 3 \%$ higher.

In the basal septum, very similar values are observed for WKY and SHR for the same age rats. Nevertheless, again an increase of $\sim 4 \%$ can be observed for the older group of animals.

Finally, in the lateral wall large variations are observed. The value for WKY rats does not seem to change significantly between 3 and 12 months and the mean is even found to be $\sim 0.5 \%$ lower. The same applies to SHR, which, even if higher than WKY for both age points $(\sim 1 \%$ and $\sim 1.5 \%)$, has a mean value that seems to also decrease by $\sim 0.5 \%$ in older rats.

\section{Discussion}

In this study we assess the deposition of collagen in WKY and SHR rats at two different time points, corresponding to 3 and 12 months old, and three different regions of the left ventricle (apex, basal septum and lateral wall). For this purpose, X-PCI tomograms $(0.65 \mu \mathrm{m}$ voxel size) of rat heart biopsies were acquired, and segmented using an optimized deep neural network.

In this way, this manuscript presents an extension of preliminary work in this field [7] using the same imaging technique, but an improved analysis method that allows automatic collagen segmentation and fraction computation thanks to trained deep neural networks. 

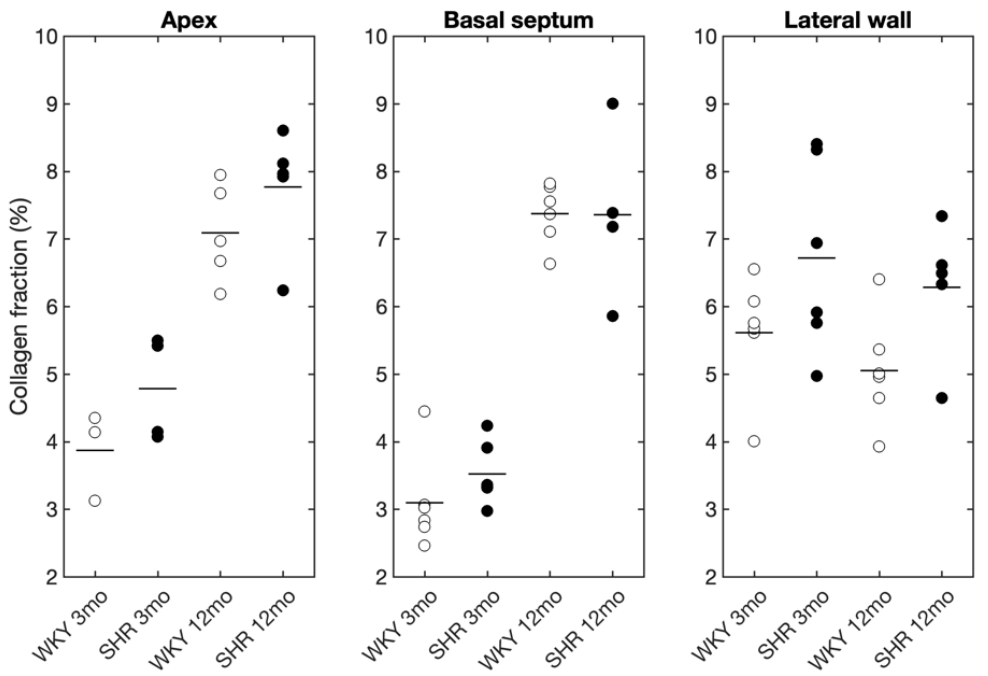

Fig. 3. Results for collagen fraction in the apex, basal septum and lateral wall of the left ventricle, respectively. Circles and full dots represent data points of control (WKY) and hypertensive model (SHR) respectively. Lines correspond to the mean value.

The results presented in Fig. 3 show a general increased tendency in collagen with age as well as in the hypertensive case. This is in accordance with the literature, since it is well-known that the collagen deposition in the extracellular matrix increases with aging. It is also known that the response of hypertensive hearts is to produce a stiffening of the myocardium (by collagen deposition) to generate higher forces and improve its pumping capabilities $[4,6,13]$.

With regards to aging, we can see that in both the apex and basal septum, the mean collagen fraction increases by $\sim 3-4 \%$ in the 12 months old cases. Nevertheless, in the lateral wall the values for the WKY remain similar or even slightly lower in the older specimens.

Looking at the differences between models, we see that in the apex there is an increase of $\sim 1 \%$ in SHR at both time points. Nevertheless, the basal septum appears to show no or very small difference in the collagen values between the models, and the lateral wall only shows an increase of $\sim 1-1.5 \%$ in SHR at both ages.

Even if lower, the presented values show a similar tendency as observed in the most recent related publication [6]. There, quantification of collagen in the lateral wall of left ventricle of these two models by using confocal microscopy and a dedicate image processing pipeline was performed. For collagen fraction, they find close values in 3 months old WKY and SHR, which, as similarly shown in Fig. 2, do not change either for 12 months old WKY. Nevertheless, they show an increase with high variability for 12 months old SHR, which is not so clear in our case even if we also observe high variance. 
The differences in values, as reviewed elsewhere [5], can be caused by many factors, mostly related to setup and sample preparation, such as imaging technique, resolution, use of staining, differences in tissue processing or collagen fraction calculation method.

The results presented in this paper have been obtained from a low number of specimens ( 2 rats per model and time point) and a few regions of interest analyzed per biopsy (3-6), which limits this study from reaching significant clinical conclusions. Moreover, during the segmentation procedure, another limitation that arises is the fact the blood cells and collagen appear to have similar characteristics, which may introduce some unwanted variations. For this reason, the selected regions of interest to analyze were chosen to have the least possible amount of visible vasculature possible.

The fact that the presented values match the well-known evolution of collagen amount as well as the most recent literature, demonstrates that X-PCI and the use of deep neural network architectures such as the optimized U-net shown here are an alternative powerful tool for the quantitative investigation of cardiac collagen matrix.

\section{Conclusion}

This study extends the assessment of collagen fraction by X-PCI to the comparison between WKY and SHR rats at 3 and 12 months old. The analysis has been performed using an optimized deep neural network, which enables automatic segmentation for unlabeled regions of interest. The results obtained follow the tendencies observed in the literature, therefore posing X-PCI as a valuable tool for the assessment and quantification of cardiac micro-structure. Nevertheless, higher statistics would be needed to attain clinical significance.

Acknowledgments We acknowledge Monica Zamora and Eduard Guasch for animal handling, and Xavier Buyse Sánchez for his support in the labelling task. In addition, we acknowledge the Paul Scherrer Institut, Villigen, Switzerland for provision of synchrotron radiation beamtime at the beamline TOMCAT (X02DA) of the Swiss Light Source. This project was supported by the grant \#2017-303 of the Strategic Focal Area "Personalized Health and Related Technologies (PHRT)", and the grant C17-04 of the Strategic Focal Area "Swiss Data Science Center (SDSC)" of the ETH Domain.

\section{References}

1. World Health Organization (2017) Cardiovascular Diseases (CVDs) Fact Sheet. http://www.who.int/en/news-room/fact-sheets/detail/cardiovasculardiseases-(cvds). Accessed 18 Jan 2019

2. Filipoiu FM (2013) Atlas of Heart Anatomy and Development. In: Springer Science \& Business Media. London.

3. Weber KT, Sun Y, Bhattacharya SK, et al (2013) Myofibroblast-mediated mechanisms of pathological remodelling of the heart. Nat. Rev. Cardiol.

4. Burchfield JS, Xie M, Hill JA (2013) Pathological ventricular remodeling: 
Mechanisms: $\quad$ Part 1 of $2 . \quad$ Circulation. https://doi.org/10.1161/CIRCULATIONAHA.113.001878

5. Schipke J, Brandenberger C, Rajces A, et al (2017) Assessment of cardiac fibrosis: a morphometric method comparison for collagen quantification. $\mathrm{J}$ Appl Physiol. https://doi.org/10.1152/japplphysiol.00987.2016

6. LeGrice IJ, Pope AJ, Sands GB, et al (2012) Progression of myocardial remodeling and mechanical dysfunction in the spontaneously hypertensive rat. AJP Hear Circ Physiol. https://doi.org/10.1152/ajpheart.00748.2011

7. Dejea H, Garcia-Canadilla P, Stampanoni M, et al (2017) Microstructural analysis of cardiac endomyocardial biopsies with synchrotron radiation-based X-ray phase contrast imaging. In: Lecture Notes in Computer Science (including subseries Lecture Notes in Artificial Intelligence and Lecture Notes in Bioinformatics)

8. Marone F, Stampanoni M (2012) Regridding reconstruction algorithm for realtime tomographic imaging. J Synchrotron Radiat. https://doi.org/10.1107/S0909049512032864

9. Paganin D, Mayo SC, Gureyev TE, et al (2002) Simultaneous phase and amplitude extraction from a single defocused image of a homogeneous object. J Microsc. https://doi.org/10.1046/j.1365-2818.2002.01010.x

10. Sommer C, Straehle C, Ullrich K, Hamprecht F a (2011) ILASTIK: INTERACTIVE LEARNING AND SEGMENTATION TOOLKIT Heidelberg Collaboratory for Image Processing ( HCI ), University of Heidelberg. Eighth IEEE Int Symp Biomed Imaging. https://doi.org/10.1109/ISBI.2011.5872394

11. Ronneberger O, Fischer P, Brox T (2015) U-net: Convolutional networks for biomedical image segmentation. In: Lecture Notes in Computer Science (including subseries Lecture Notes in Artificial Intelligence and Lecture Notes in Bioinformatics)

12. Wong KCL, Moradi M, Tang H, Syeda-Mahmood T (2018) 3D segmentation with exponential logarithmic loss for highly unbalanced object sizes. In: Lecture Notes in Computer Science (including subseries Lecture Notes in Artificial Intelligence and Lecture Notes in Bioinformatics)

13. Horn MA, Trafford AW (2016) Aging and the cardiac collagen matrix: Novel mediators of fibrotic remodelling. J. Mol. Cell. Cardiol. 